\title{
Performance Assessment of DFT-OFDM and DWT-OFDM Systems in the Presence of the SSPA and Fading Channel
}

\author{
Abdolreza Kiani ${ }^{1}$ and Soheila Mousavi ${ }^{2}$ \\ ${ }^{1,2}$ Department of Electrical Engineering, Abadan Branch, Islamic Azad University, Abadan, Iran \\ E-mail:'1ab2rezakiani@gmail.com, 2sso.mousavi@gmail.com
}

\begin{abstract}
This paper investigates performance degradation of conventional Orthogonal Frequency Division Multiplexing (OFDM) and Discrete Wavelet Transform based OFDM (DWT-OFDM) systems when the signals are passed through a nonlinear High Power Amplifier (HPA) and fading channel. In the case of DWT-OFDM, several wavelets such as Daubechies, Symlet and Biorthogonal are evaluated. Simulation results in terms of PAPR, PSD, and Bit Error Rate (BER) show that DWT-OFDM is more robust against interference and multipath effects compared to DFT-OFDM, and increasing the length of the basis function improves BER and PAPR.
\end{abstract}

Keywords: DFT-OFDM; DWT-OFDM; BER; PAPR; High Power Amplifier.

\section{INTRODUCTION}

Multicarrier Modulation (MCM) is an efficient modulation scheme which divides the incoming high rate data into lower rate data. The duration of symbols is increased by simultaneously transmitting $N$ data symbols which leads to robustness against channels fading, impulsive noise, and Inter Symbol Interference (ISI).

OFDM is a multicarrier scheme commonly used nowadays. OFDM has been widely adopted and standardized across the world. A number of applications and standards which use OFDM include Digital Audio Broadcasting (DAB), Digital Video Broadcasting (DVB), WiFi (IEEE $802.11 \mathrm{a} / \mathrm{g} / \mathrm{j} / \mathrm{n})$, World Wide Interoperability for Microwave Access (WiMAX-IEEE 802.16), Ultra Wide Band Wireless Personal Area Network (UWB Wireless PAN-IEEE 802.15.3a) and Mobile Broadband Wireless Access (MBWA-IEEE802.20). Inverse Fast Fourier Transform (IFFT) and FFT are used in OFDM to multiplex the signals together and demultiplex the signals in the receiver, respectively [1]. A Cyclic Prefix (CP) is prepended to data signals before transmission. The purpose of the $\mathrm{CP}$ is to minimize ISI (Inter-symbol interference). However, the CP has disadvantages such as reducing the spectral containment of the channel, power consumption, etc. [2].
Wavelet transformation has recently emerged as a strong candidate for digital communications [3]. In DFT-OFDM systems, signals only overlap in the frequency domain while DWT-OFDM signals overlap both in the time and frequency domains, so there is no need for the CP as in the DFT-OFDM case. Therefore, by using this transformation, the spectral containment of the channel is improved [4].

Performance of MCM communication systems is highly sensitive to nonlinear distortions arising mainly from the HPA [5-7]. To achieve more output power, transmission power should be increased, which in turn causes the HPA to operate in saturation region. Hence, it seems necessary to assess and compare the DFT-OFDM and DWTOFDM system performances in the presence of the HPA.

This paper aims to evaluate the impact of the distortion introduced by the nonlinear behavior of a Solid State Power Amplifier (SSPA), as an HPA, which is commonly used in cellular systems. In this study, the Rapp model is used both in DFT-OFDM and DWT-OFDM systems. The Rapp model is characterized by [8]:

$F A_{M / A M}=V_{\text {in }} /\left[1+\left(V_{\text {in }} / V_{\text {sat }}\right)^{2 p}\right]^{1 / 2 p}$

Where $V_{i n}$ is the magnitude of the input signal, $p$ is smoothness factor, $F A_{M / A M}$ is the magnitude of 
the output signal, and $V_{\text {sat }}$ is the output saturation level. The smoothness factor controls transition for the amplitude gain as the output amplitude approaches saturation. Fig. 1 shows input-output characteristics for various smoothness factors $p$. Also, the phase transfer function is almost zero.

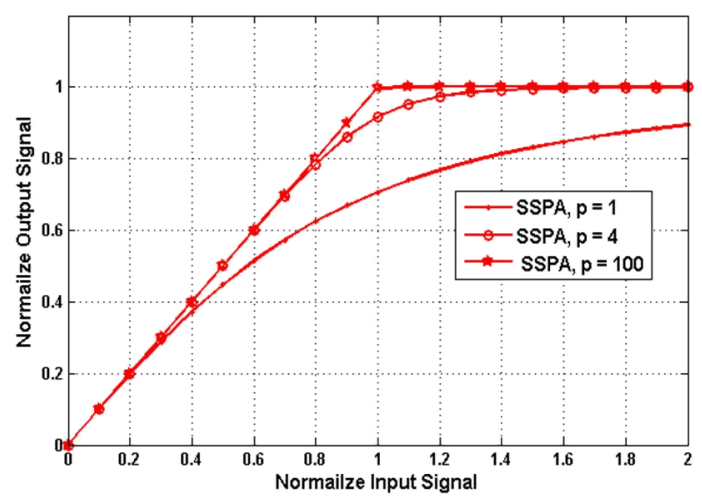

Fig. 1. Input-output characteristic of the Rapp model.

The paper is organized as follows. Section II introduces block diagrams of DFT-OFDM and
DWT-OFDM systems, respectively. Section III considers the PAPR (peak to average power ratio) performance in DFT-OFDM and DWT-OFDM systems. Section IV and V evaluate the Bit Error Rate (BER) performance without SSPA, in the presence of fading channel and with SSPA, respectively. Finally VI concludes the paper.

\section{BLOCK DIAGRAMS OF DFT-OFDM AND DWT-OFDM SYSTEMS}

DFT-OFDM and DWT-OFDM transceiver systems are shown in Fig. 2. In DFT-OFDM, the data bit-stream is first mapped onto QAM constellation to form a complex symbol followed by a $\mathrm{S} / \mathrm{P}$. Then it is modulated onto orthogonal subcarriers using IDFT. After $\mathrm{P} / \mathrm{S}$, a $\mathrm{CP}$ (that is $25 \%$ of each symbol in practical systems) is wrapped to the symbols. Then the signals are passed through the HPA followed by channel. At the receiver, the $\mathrm{CP}$ is discarded. The resulting signal is demodulated to recover the original data bits.

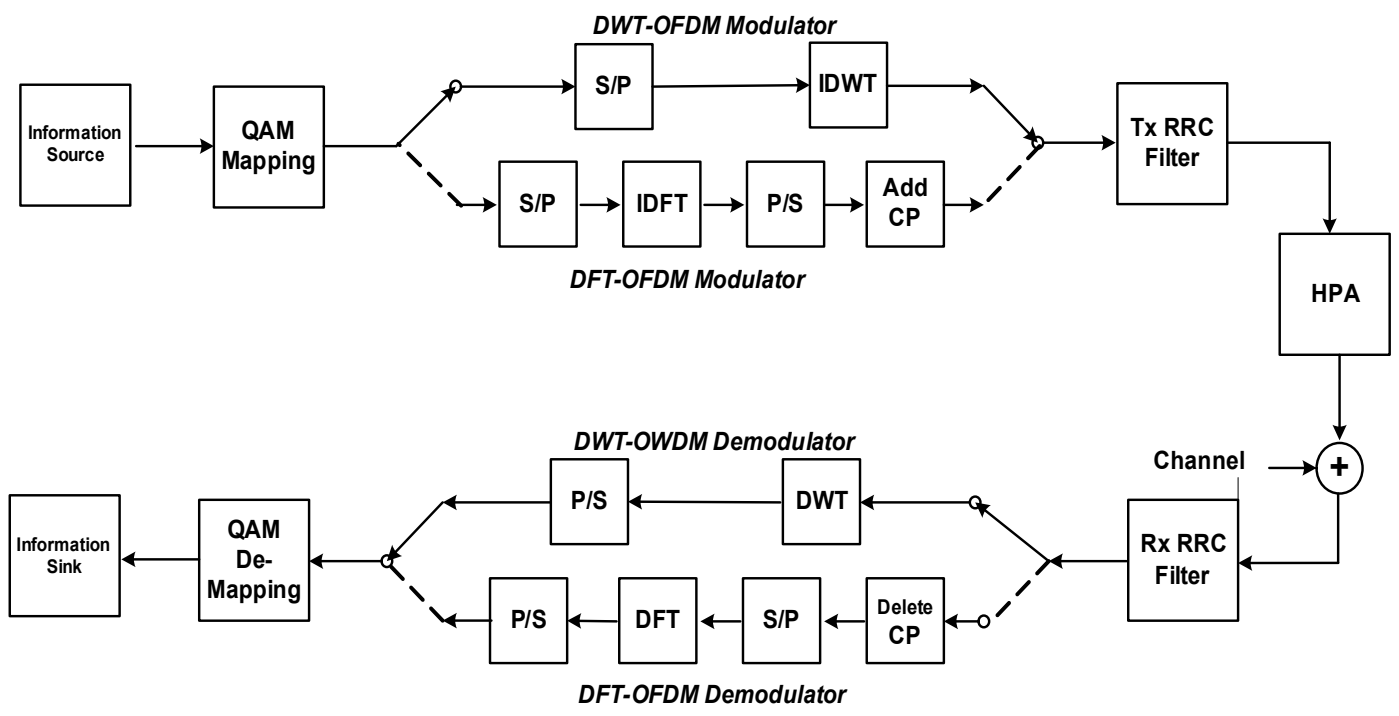

Fig. 2. DFT-OFDM and DWT-OFDM transceiver block diagrams. 


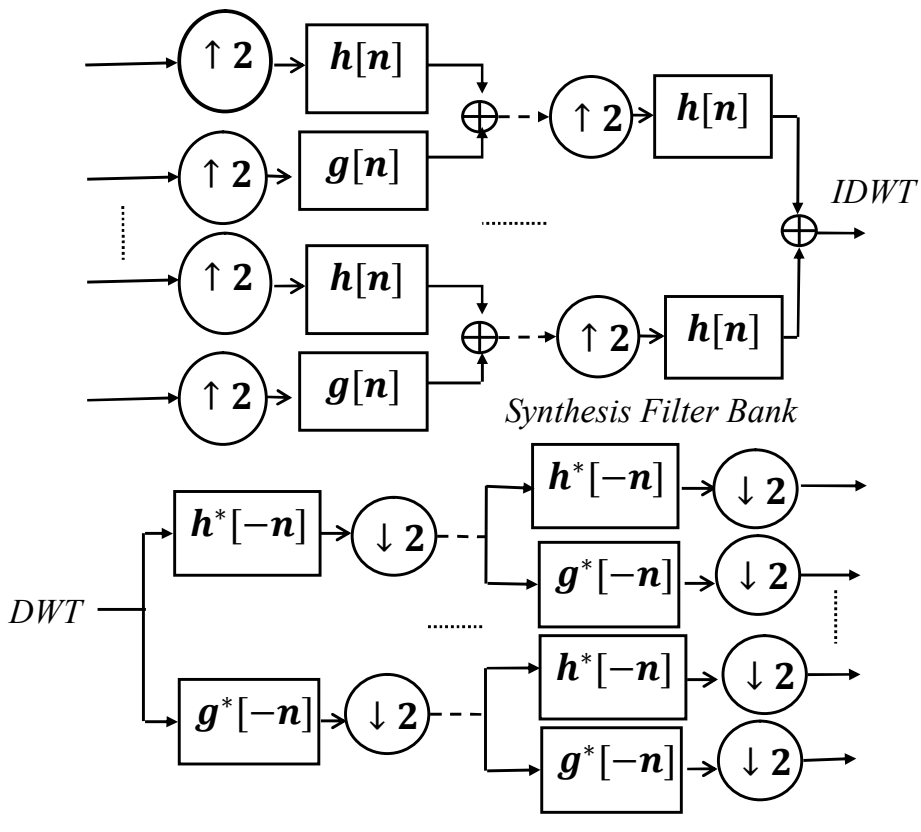

Analysis Filter Bank

Fig. 3. IDWT and DWT blocks.

Wavelet Transform (WT) is a class of generalized Fourier transforms with basis function being localized well both in the time and frequency domains. They are constructed by means of Quadrature Mirror Filter (QMF) pairs [9-10]. It has been shown that DWT-OFDM is more robust to narrowband interference and multipath propagation loss than DFT-OFDM [11]. In DWT-OFDM transmitter, the incoming signal is first converted from serial to parallel. In the case of DWT-OFDM, the number of iterations can be expressed by:

Number of Iteration $=\log _{2}$ (Number of Subcarriers)

Fig.3 shows DWT and inverse DWT (IDWT) blocks. IDWT (as the synthesis filter bank) and DWT (as the analysis filter bank) are used in place of IDFT and DFT, respectively, at the transmitter and receiver. Any iteration of IDWT upsamples two signals and filters one with a High Pass (HP) Finite Impulse Response (FIR) filter and the other one with a Low Pass (LP) FIR filter. The outputs of the HP and LP filters are then subsequently added [12]. Consequently, DWT-OFDM does not require $\mathrm{P} / \mathrm{S}$ in the transmitter and $\mathrm{S} / \mathrm{P}$ in the receiver. In our study, several wavelets such as dbN, symN, biorNr, $\mathrm{Nd}$ are evaluated. When analysis bank is exchanged with the synthesis bank, the system will be still a perfect reconstruction (PR) [13]. Accordingly, if these wavelets preserve orthogonality between the symbols, it is expected that the Bit Error Rare (BER) plot lies on the theoretical BER plot.

Fundamentally, DFT-OFDM and DWT-OFDM have many similarities as both use orthogonal waveforms as subcarrier. The main difference between DFT-OFDM and DWT-OFDM lies on the shape of the subcarrier and in the way they are created. One important property of wavelet is that the waveforms being used in general are longer than the transform duration of each symbol [14-15]. This causes DWT-OFDM symbols to overlap in the time domain.

The multicarrier symbols of DFT-OFDM do not overlap each other as IDFT and DFT transforms are carried out for each group of subcarriers independently. The use of longer waveforms in DWT-OFDM, on the other hand, allows better frequency localization of subcarriers while in DFTOFDM the rectangular shape of the DFT window generates large side lobes [16].

Simulation parameters and characteristics of wavelet families are shown in Tables I and II, respectively. For a fair comparison, the $\mathrm{CP}$ is not used for DFT-OFDM in AWGN channel, but we used $\% 25$ of subcarrier length for $\mathrm{CP}$ in fading channel. 
A. Kiani and S. Mousavi / International Journal of Computer Networks and Communications Security, 1 (7), December 2013

\begin{tabular}{|c|c|c|c|}
\hline \multirow{6}{*}{\multicolumn{4}{|c|}{$\begin{array}{lc}\text { Number of DWT/DFT Point } & 64 \\
\text { Length of the CP for AWGN } & 0 \\
\text { Length of the CP for DWT-OFDM } & 0 \\
\text { Length of the CP for DFT-OFDM in Fading Channel } \% 25 \\
\text { Data Modulation } \\
\text { Shaping Filter RRC }(\alpha=0.22 \text {, Over sampling Rate }=4)\end{array}$}} \\
\hline & & & \\
\hline & & & \\
\hline & & & \\
\hline & & & \\
\hline & & & \\
\hline \multicolumn{4}{|c|}{ Table II Characteristics of Wavelet Family } \\
\hline Name & Length & Vanishing moment & orthogonal \\
\hline $\mathrm{dbN}$ & $2 \mathrm{~N}$ & $\mathrm{~N}$ & Yes \\
\hline symN & $2 \mathrm{~N}$ & $\mathrm{~N}$ & Yes \\
\hline bior3.5 & (12.4) & 3 (dec.) & No \\
\hline bior5.5 & $(9.11)$ & $5(\mathrm{dec})$. & No \\
\hline
\end{tabular}

Figs.4a-e illustrate the spectra of 8 adjacent subcarriers for DFT-OFDM and DWT-OFDM with $\mathrm{db} 4, \mathrm{db} 8$ and bior5.5 filters, respectively. By increasing the length of filters in Daubechies family, the bandwidth (BW) of each subcarrier is decreased (Fig. 4-b and Fig. 4-e). The shapes of bior5.5 subcarriers exhibit the unfulfilled orthogonal condition between the HP and LP filters. More details about the system can be seen in [17-18].

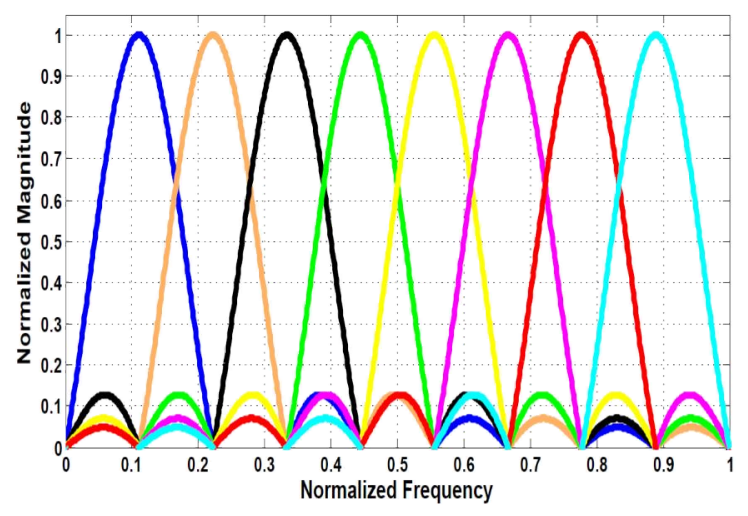

Fig. 4-a. Spectra of 8 DFT-OFDM subcarriers.

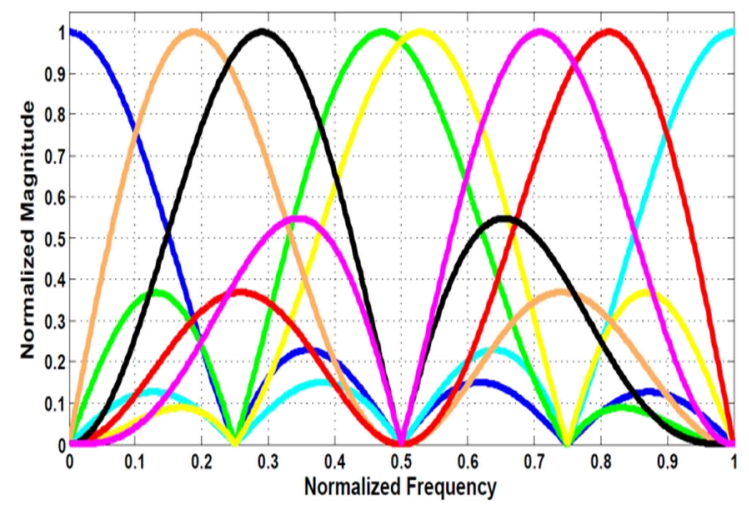

Fig. 4-b. Spectra of 8 DWT(db1)-OFDM subcarriers.

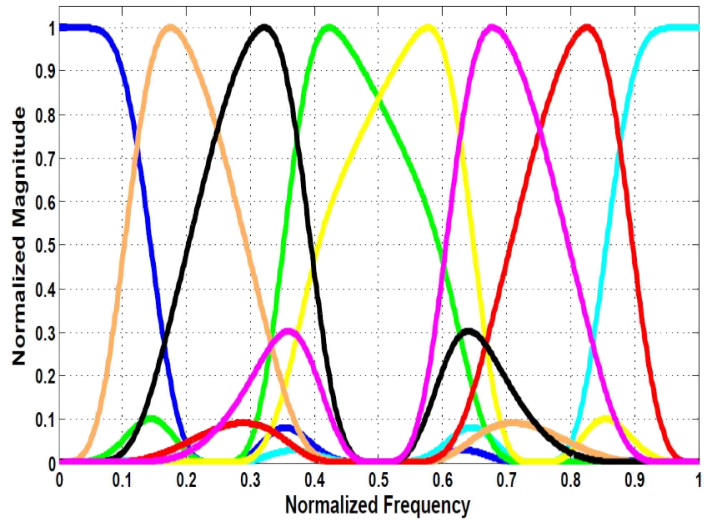

Fig. 4-c. Spectra of 8 DWT(db4)-OFDM subcarriers.

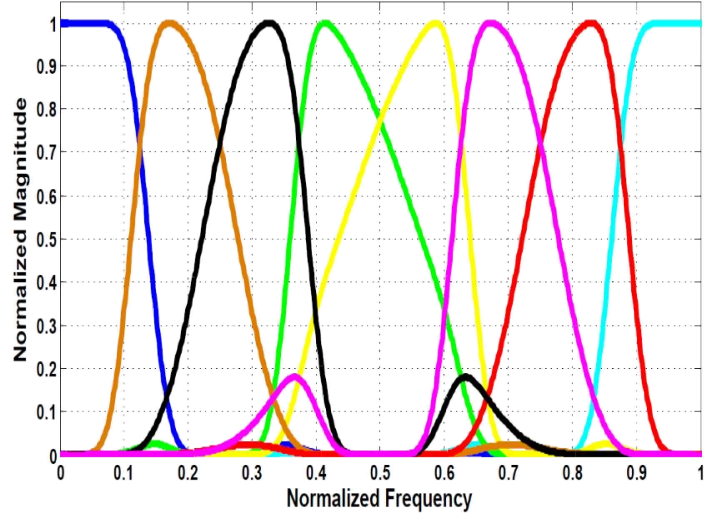

Fig. 4-d. Spectra of 8 DWT(db8)-OFDM subcarriers

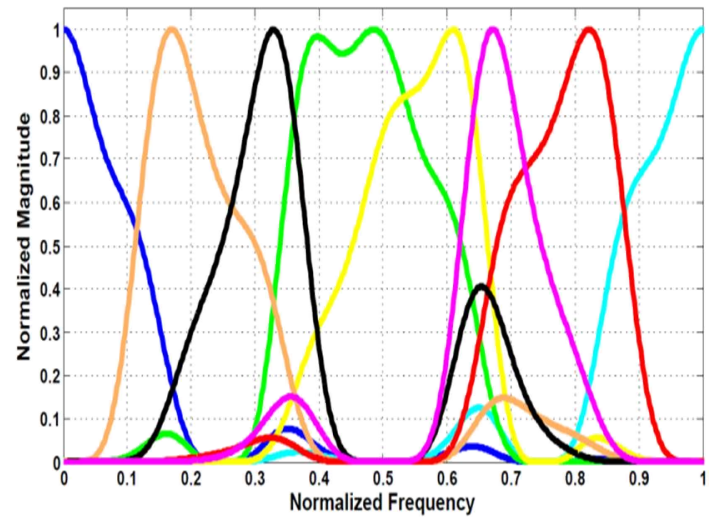

Fig. 4-e. Spectra of 8 DWT(bior5.5)-OFDM subcarriers.

\section{PAPR IN DFT-OFDM AND DWT-OFDM SYSTEMS}

One of main drawbacks of OFDM is its high PAPR. Signals with large peaks may be obtained as a result of constructive superposition of subcarriers. PAPR is defined as the ratio between the maximum power occurring in OFDM symbol to the average power of the same OFDM symbol: 


$$
P A P R=\max |x(t)|^{2} / E\left[|x(t)|^{2}\right]
$$

Where E[.] denotes expectation. PAPR depends linearly on the number of subcarriers, but in systems with a large number of subcarriers, the probability of a symbol with a large PAPR is small and vice versa. This leads to use CDF (Cumulative Distribution Function) to describe PAPR distribution. High peak power is a disadvantage of HPAs. Due to amplifier imperfection, peaks are distorted nonlinearly. The result can be interpreted as an ICI (inter-carrier interference) in the system. In general, PAPR is evaluated from the discrete time samples by oversampling. PAPR can take values in a range that is proportional to the number of subcarriers. In this study, the DFT-OFDM and DWT-OFDM schemes with 64 subcarriers, each modulated with QPSK, were compared in terms of CDF. Fig. 5 shows that while DWT(db4)-OFDM has a comparable PAPR performance with DFT-OFDM, other wavelets exhibit inferior performance in comparison to DFT-OFDM.

As a rectangular window in DFT-OFDM has large spectral sidelobes, there is a large spectral leakage. This could pose a problem in some applications. For example, in some wire systems that transmission of the PSD of downstream signal needs to fall below a threshold in the frequency bands of upstream transmission to avoid interference, the PSD should be attenuated in amateur radio bands to allow egress emission control. Fig. 6 shows that Sym 8 and db8 can reduce the spectral leakage more than DFT-OFDM.

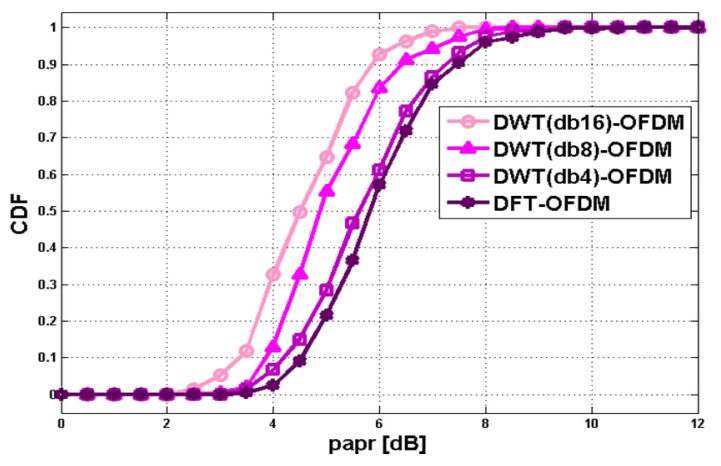

Fig. 5. CDFs of the PAPR for different schemes.

\section{SIMULATION WITHOUT POWER AMPLIFIER}

As for sanity check, performance of both DFTOFDM and DWT-OFDM systems without SSPA are evaluated in AWGN channel. Fig. 7 shows the BER performance of the QPSK modulation scheme in an AWGN channel. It can be observed in this
Figure that the BER performances of DWT-OFDM and DFT-OFDM are the same except for bior3.5, and bior5.5. These wavelets are not orthogonal and thus the orthogonality between subcarriers is destroyed. The difference between $\mathrm{dbN}$ and symN is not significant, because they do not use any nonlinear element such as HPA and the model is perfectly reconstructive. This validates the simulations. Fig 8 shows performance of DFTOFDM and DWT-OFDM in 15 Tap fading channel. In this Fig DWT(db8-Sym4)-OFDM are better performance in comparison to DFT-OFDM. The significant point is we don't use CP in DWTOFDM but there are $25 \%$ in DFT-OFDM. The lack of $\mathrm{CP}$ will result in less power consumption. These simulations were carried out using the block-type (time-multiplexed) pilot-aided channel estimation in fading channel. This is due to this fact that the block-type pilot arrangement has been developed under the assumption of slow fading channel, while the other basic arrangement i.e. the comb-type (frequency-multiplexed) has been introduced for equalizing the fast fading channels [20]. In this study, the information signal is impaired by the time-invariant (nonlinear) behavior of the HPA rather than its temporal variations. In Fig. 9 pilotchannel estimation in fading channel is used for QPSK modulation and compensated phase and amplitude of the received signal.

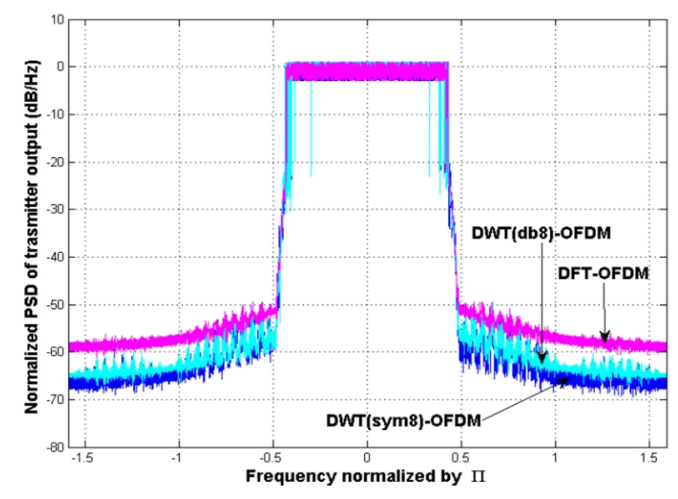

Fig. 6. PSD comparison of DWT(db8)-OFDM, $D \overline{W T}(\operatorname{sym} \overline{8})=O F D M$ and $D F T=O F D M$.

\section{RESULTS IN THE PRESENCE OF THE SSPA}

An HPA is usually identified by two parameters known as Input Back Off (IBO) and Output Back Off (OBO), defined in decibel as:

$I B O=10 \log _{10}\left(P_{\text {imax }} / P_{i}\right)$ and $O B O=10 \log _{10}$ $\left(P_{\text {omax }} / P_{o}\right)$, respectively, where $P_{\text {imax }}$ and $P_{\text {omax }}$ are the mean power of the input and output signals of 
the HPA. $P_{\text {omax }}$ is the maximum output power (saturation power), and $P_{\text {imax }}$ is the input power corresponding to the maximum output power [19]. A pictorial description of $\mathrm{OBO}$ and IBO is shown in Fig. 10 and defined (on a logarithmic scale) as the difference between the maximum output power and the output power at the quiescent point. Fig.11 shows the BER performance of DFT-OFDM and DWT-OFDM when Rapp model is applied with smoothness factor $p=1$ at $\mathrm{OBO}=3 \mathrm{~dB}$. In this Figure DWT-OFDM outperforms DFT-OFDM. As shown in Fig.11, by decreasing the order of Daubechies filters, performance of DWT-OFDM system will be degraded. This behavior is more obvious at $E_{b} / N_{o}$ values larger than $20 \mathrm{~dB}$.

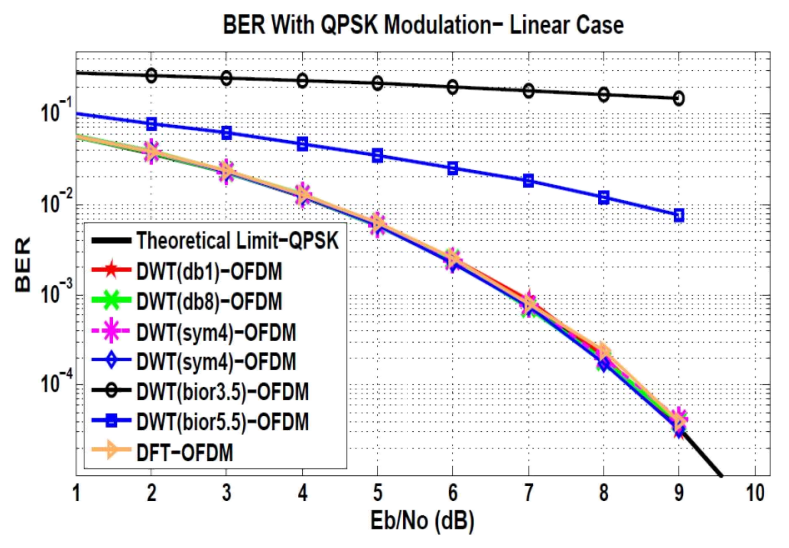

Fig. 7. Performance of DFT-OFDM and DWT-OFDM for the linear case in AWGN channel.

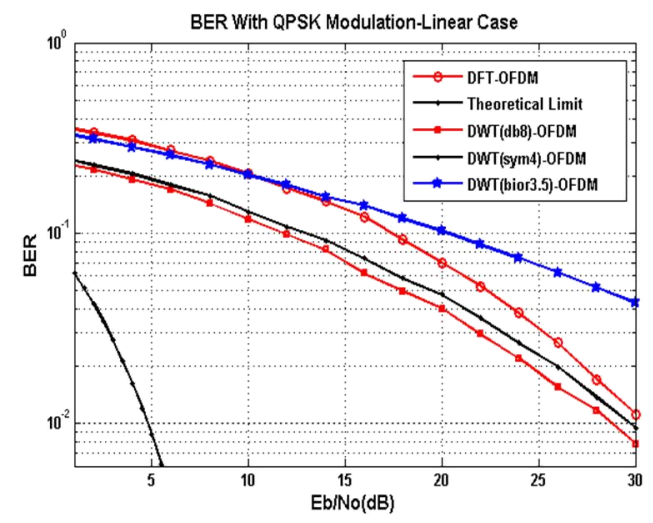

Fig. 8. Performance of DFT-OFDM and DWT-OFDM for the linear case in 15 Tap Fading channel.

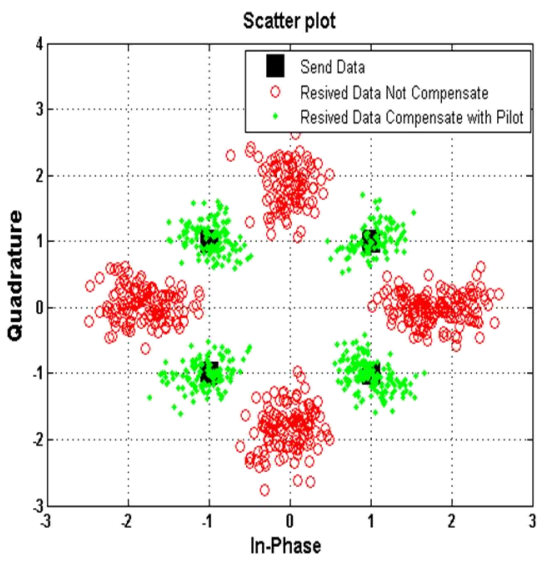

Fig. 9. Channel estimation with block-type pilot.

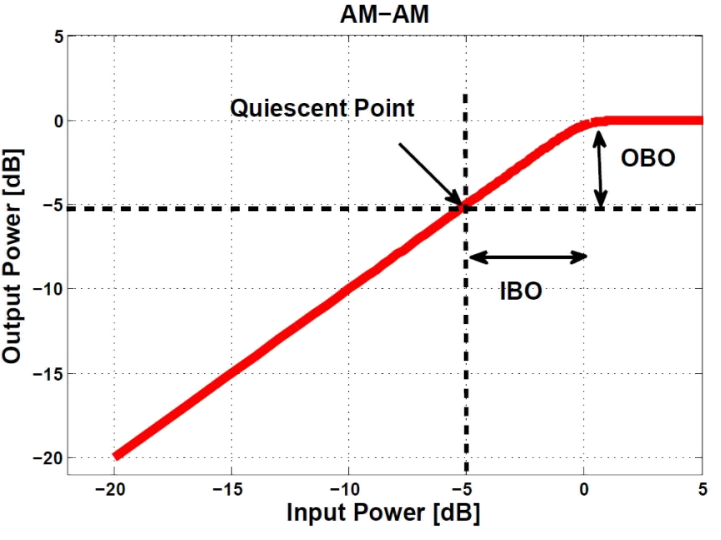

Fig. 10. AM-AM characteristic.

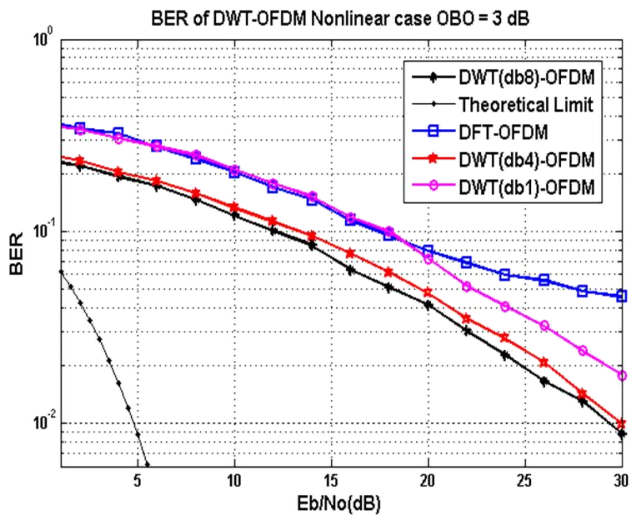

Fig. 11. Performance of DFT-OFDM and DWT-OFDM in the presence of SSPA and fading channel. 
6

\section{CONCLUSIONS}

In this paper the BER, PAPR performances of DFT-OFDM and DWT-OFDM in the presence of SSPA and fading channel were evaluated. According to simulation results, it was found that the BER performance of DWT-OFDM is the same as DFT-OFDM in AWGN channel for the linear system i.e. without SSPA as a nonlinear block. As the filter length was altered among the members of the same family (except for the biorthogonal family), no perceivable difference was observed in the system performance. However, if Rapp model is applied, this difference becomes significant. In Daubechies and Symlet families, when the length of the filter decreased, the BER and TD performances were degraded. The above results were confirmed for the corresponding equalized schemes as well. The advantages of DWT-OFDM can be summarized as follows:

1) Due to lack of CP, DWT-OFDM reducing the spectral containment of the channel, power consumption, etc.

2) In DFT-OFDM systems, signals only overlap in the frequency domain while DWT-OFDM signals overlap both in the time and frequency domains.

3) DWT-OFDM does not require $P / S$ in the transmitter and $S / P$ in the receiver.

4) DWT-OFDM can created subcarrier with different shapes.

5) Wavelets exhibit inferior performance in $P A P R, B E R$ and PSD in comparison to DFTOFDM.

\section{REFERENCES}

[1] S.Weinstein, P. Ebert, "Data transmission by frequencydivision multiplexing using the discrete Fouriertransform," IEEE Trans. on Communications, COM-19, 5:628-634, Oct 1971.

[2] K. Abdullah, Z. M. Hussain, "Studies on DWT-OFDM andFFT-OFDM systems," International Conference on Communication, Computer and Power (ICCCP'09),MUSCAT, pp. 15-18,Feb 2009.

[3] M. K. Lakshmanan and H. Nikookar, "A review of wavelets for digital wireless communication,"Journal on Wireless Personal Communication, vol. 37, no.3-4, pp. 387420,Springer, May 2006.

[4] R. Dilmirghani, M. Ghavami, "Wavelet Vs FourierbasedUWB systems,"18th IEEE International Symposium on Personal, Indoor and Mobile Radio Communications, pp.1-5, Sep 2007.

[5] E. Costa, S. Pupolin, "M-QAM-OFDM system performance in the presence of a nonlinear amplifier and phase noise,'IEEE Trans. on Communications, vol. 50, no.3, Mar 2002.

[6] V.A. Bohara, and S.H. Ting, "Theoretical analysis of OFDM signals in nonlinear polynomial models," 6thInternational Conference on Information, Communications\&Signal Processing, pp. $1-5$, 10-13 Dec. 2007.

[7] N.Y. Ermolova, "Nonlinear amplifier effects on clipped filtered multicarrier signals," IEEE Proc. Communication, vol.153,no.2, pp.213218 Apr. 2006.

[8] C. Rapp, "Effects of HPA-nonlinearity on a 4DPSK/OFDM-signal for a digital sound broadcasting system, "in Proceedings of the Second European Conference on Satellite Communications, pp. 179-184,Liege, Belgium, Oct. 22-24, 1991.

[9] A. R. Lindsey, "Wavelet packet modulation: a generalized method for orthogonally multiplexed communications," in IEEE 27thSoutheastern Symposium on System Theory, pp. 392-396, 1995.

[10]D. Daly, C. Heneghan, A. Fagan, and M. Vetterli, "Optimal wavelet packet modulation under finite complexityconstraint," in Proc. ICASP, vol. 3, pp. 2789-2792,2002.

[11]P. Kaewmanee, R. F.Ormondroyd, C. R. Walters, "Resilient adaptive wavelet packet modulation scheme for Used in Time and Frequency Selective Channels Using the BestTree Search Algorithm,'2004 IEEE Military Communications Conference, pp. 1566-1571, Milom, 2004.

[12]P.P.Vaidyanathan, "A theory for multiresolutionsignaldecomposition: The wavelet representation,'IEEETrans.Pattern Anal. Machine Intell, vol.11, pp.674-693, Jul 1989.

[13] G.Strang, T.Nquyen, Wavelets and Filter Banks, Wellesley-Cambridge Press, 1996.

[14]M.Vetterli, J. Kovacevic, Wavelets and Subband Coding.Prentice HallPTR, Englewood Cliffs, New Jersey, 1995.

[15]I.Daubechies, Ten Lectures on Wavelets, Philadelphia:SIAM, 1992.

[16] D.Karamehmedović, M.K.Lakshmanan, H.Nikookar, "Performance evaluation of WPMCM with carrier frequency offset and phase noise", Journal of Communications, vol. 4, no. 7, pp. 496-508, Aug 2009. 
A. Kiani and S. Mousavi / International Journal of Computer Networks and Communications Security, 1 (7), December 2013

[17]H. Nikookar and M.K.Lakshmanan, "Comparison of sensitivity OFDM and wavelet packet modulation to time synchronization error," The 19th Annual IEEEInternational Symposium on Personal, Indoor and Mobile Radio Communications (PIMRC'08), 2008.

[18]M. Gautier, M. Arndit, J. Lienard, "Efficient wavelet packet modulation for wireless communication," The Third Advanced International Conference onTelecommunications (AICT'07), pp. 278284, Apr 2007.

[19] H. Zareani, V. T. Vakili, "Analysis of EVM, BER, TD performance of the OFDM system in the presence ofjointly nonlinear distortion," Institute TELCOM andSpringerVerlag, France, May 2009.

[20] R. Van Nee and R. Prasad, OFDM for Wireless Multimedia Communications. Artech House, 2000. 